\title{
Combination OX40 agonism/CTLA-4 blockade with vaccination reverses anergy and primes tumor-specific CD8 T cells in mice with spontaneous prostate cancer
}

\author{
Stefanie N Linch*, Melissa J Kasiewicz, William L Redmond \\ From Society for Immunotherapy of Cancer 28th Annual Meeting \\ National Harbor, MD, USA. 8-10 November 2013
}

Targeted immunotherapy, such as anti-CTLA-4 and anti-PD-1, has proven effective in treating cancer patients. However, despite these advances, cancer remains the second leading cause of death in the US. More effective strategies designed to maximize antitumor CD8 $\mathrm{T}$ cell responses are necessary to sustain long-term immunity. Agonist anti-OX40 and antagonist anti-CTLA-4 mAb augment the CD8 $\mathrm{T}$ cell response through different mechanisms. Therefore, we investigated the additive effects of these modalities on CD8 $\mathrm{T}$ cell responses. Combination anti-OX40/anti-CTLA-4 therapy more effectively primed antigen-specific CD8 $\mathrm{T}$ cells through enhanced expansion ( $82 \%$ of circulating CD8 T cells) compared to anti-OX40 $(61 \% ; \mathrm{P}<0.05)$ or anti-CTLA-4 $(41 \% ; \mathrm{P}<0.01)$ alone. Dual therapy also drove expansion of the polyclonal CD8 population. Moreover, combination therapy induced more proliferation (Ki-67) and differentiation (granzyme B, CD127, KLRG-1) following priming, suggesting potent additive effects of these modalities. However, it is known that tumors can induce $\mathrm{T}$ cell anergy, thereby limiting an effective anti-tumor response. We tested whether combination therapy could overcome anergy using a mouse model in which mice are tolerant to membrane-bound OVA. Combination anti-OX40/anti-CTLA-4 therapy was uniquely capable of driving robust expansion $(12 \%$ of total CD8 in the spleen, vs. $2.5 \%$ anti-OX40, $\mathrm{P}<0.05$, or $1 \%$ anti-CTLA- $4, \mathrm{P}<0.01$ ) of anergic CD8 T cells, along with enhanced Ki-67 and granzyme B. To further drive expansion of antigen-specific CD8 $\mathrm{T}$ cells following therapy, we examined several methods of vaccination using nanoparticles, a Listeria monocytogenes vector, or anti-DEC-205 mAb (all conjugated to OVA) in the presence of anti-CD40. In anergic mice given combination therapy, vaccination with anti-DEC-205OVA/anti-CD40 produced a 9-fold expansion of OT-I CD8 $\mathrm{T}$ cells compared to soluble OVA alone, greater than all other vectors combined. We next tested the efficacy of combination therapy with vaccination on anergy in a spontaneous model of prostate cancer. Combination therapy with vaccination drove expansion (2-fold over monotherapy, $\mathrm{P}<0.05$ ) and differentiation (granzyme $\mathrm{B}$, KLRG-1, CD25) of both anergic tumor-specific and polyclonal CD8 $\mathrm{T}$ cells. Importantly, dual therapy with vaccination enhanced the frequency and number of IFN- $\gamma$, TNF- $\alpha$, and IL- 2 producing CD8 T cells. These data indicate that anti-OX40/anti-CTLA-4 combination therapy with vaccination can uniquely augment and drive a robust CD8 $T$ cell response capable of overcoming anergy.

Published: 7 November 2013 